\title{
Reconstruction of Tungsten (111) and (211) Surfaces Induced by Carbon and Oxygen
}

\author{
Z. Szczudlo, S. Zuber, A. Szczepkowicz* And A. Ciszewski \\ Institute of Experimental Physics, University of Wrocław \\ pl. Maxa Borna 9, 50-204 Wrocław, Poland
}

(Received July 8, 2002)

\begin{abstract}
Scanning tunneling microscopy is used to obtain images of reconstructed $W(111)$ and $W(211)$ surfaces. The reconstruction is induced by submonolayer coverages of carbon and oxygen.
\end{abstract}

PACS numbers: $68.35 . \mathrm{Bs}, 07.79 . \mathrm{Cz}$

\section{Introduction}

The (111) surface of tungsten has recently attracted some interest because of its thermal faceting properties [1-4]. The W(111) surface is an atomically rough, loosely-packed surface (in contrast to the close-packed surfaces like W(110) and $\mathrm{W}(100)$ ), which undergoes large-scale topographical reconstruction if it is annealed in the presence of various adsorbates $(\mathrm{Pt}, \mathrm{Pd}, \mathrm{Rh}, \mathrm{Ir}, \mathrm{Au}, \mathrm{O}, \mathrm{Cl})[2]$.

It is interesting that so far there exist no published images of clean W(111) surface obtained using scanning tunneling microscopy (STM), although images of $\mathrm{W}(111)$ covered by adsorbates have been published $[1,2,5]$. In this work we have attempted to supply the missing data by obtaining a high resolution STM image of W(111). Although we have not succeeded in removing all of the impurities from the tungsten surface, we have reduced the contamination coverage to a submonolayer level, and obtained a high resolution image of the surface.

*corresponding author; e-mail: asz@ifd.uni.wroc.pl 


\section{Experimental}

Observations were carried out using a commercial scanning tunneling microscope: Omicron VT STM $100 \mathrm{RH} / \mathrm{DH}$, equipped with an Auger electron spectroscopy (AES) setup. The base pressure of the vacuum system was lower than $1 \times 10^{-10}$ torr. Home made tungsten scanning tips were used; they were prepared by electrochemical etching in a solution of KOH. Surface scanning was carried out at room temperature, in the constant current mode. The tungsten (111) sample was cleaned in situ by repeated alternative annealing in vacuum or under the oxygen pressure of $1 \times 10^{-7}$ torr. The image processing and height profile analysis were performed using WSxM software from Nanotec [6].

\section{Results and discussion}

After the cleaning procedure has been carried out, the sample was characterised by Auger electron spectroscopy. The Auger spectrum (Fig. 1a) is dominated by a tungsten substrate signal at $179 \mathrm{eV}$. The surface is slightly contaminated by carbon (peak energy at $272 \mathrm{eV}$ ) and oxygen (peak energy at $510 \mathrm{eV}$ ). The relative AES signals of contaminants (compared to the tungsten signal) are $\frac{1}{6}$ for carbon and $\frac{1}{9}$ for oxygen.

Following AES characterisation, the sample was subjected to annealing at $1500 \mathrm{~K}$ for $3 \mathrm{~min}$ to allow for local equilibration of the atomic structure at the surface. The resulting surface structure is shown in Fig. $1 \mathrm{~b}$ and c. The reconstruction pattern follows the three-fold symmetry of W(111), but the elementary cell shown in (c) is 6 times larger compared to the unreconstructed W(111). This $6 \times 6$ reconstruction is accompanied by relatively large height corrugation $(\sim 0.25 \mathrm{~nm})$, as can be seen in Fig. 1d. This corrugation is greater than the typical corrugation $(<0.1 \mathrm{~nm})$ of unreconstructed metal surfaces [7].

Images shown in Fig. $1 \mathrm{~b}$ and $\mathrm{c}$ represent a typical pattern found on our W(111) sample. However, in one region we found a reconstructed pattern with a two-fold symmetry (Fig. 1e and f). This pattern resembles a clean W(211) surface [8], except that the distance between the atomic rows is doubled: $0.9 \mathrm{~nm}$ (instead of $0.45 \mathrm{~nm}$ for unreconstructed $\mathrm{W}(211)$ ). It appears that in this special region of the sample facets of (211) orientation appear on the surface. The angle between (111) and (211) planes is $19^{\circ} 28^{\prime}$; the appearance of (211) facets on a (111) sample may be due to a local defect, e.g. a scratch, which causes local deflection from the (111) direction. It should be noted that the image shown in Fig. If may be interpreted either as a $2 \times 1$ reconstruction, or a $2 \times 2$ reconstruction, because in our experiment we could not resolve individual atoms within the atomic rows. The height corrugation in the reconstructed (211) region is small: $0.03 \mathrm{~nm}$; however, the precise value of such a small corrugation may depend on a specific atomic configuration of the scanning tip. 

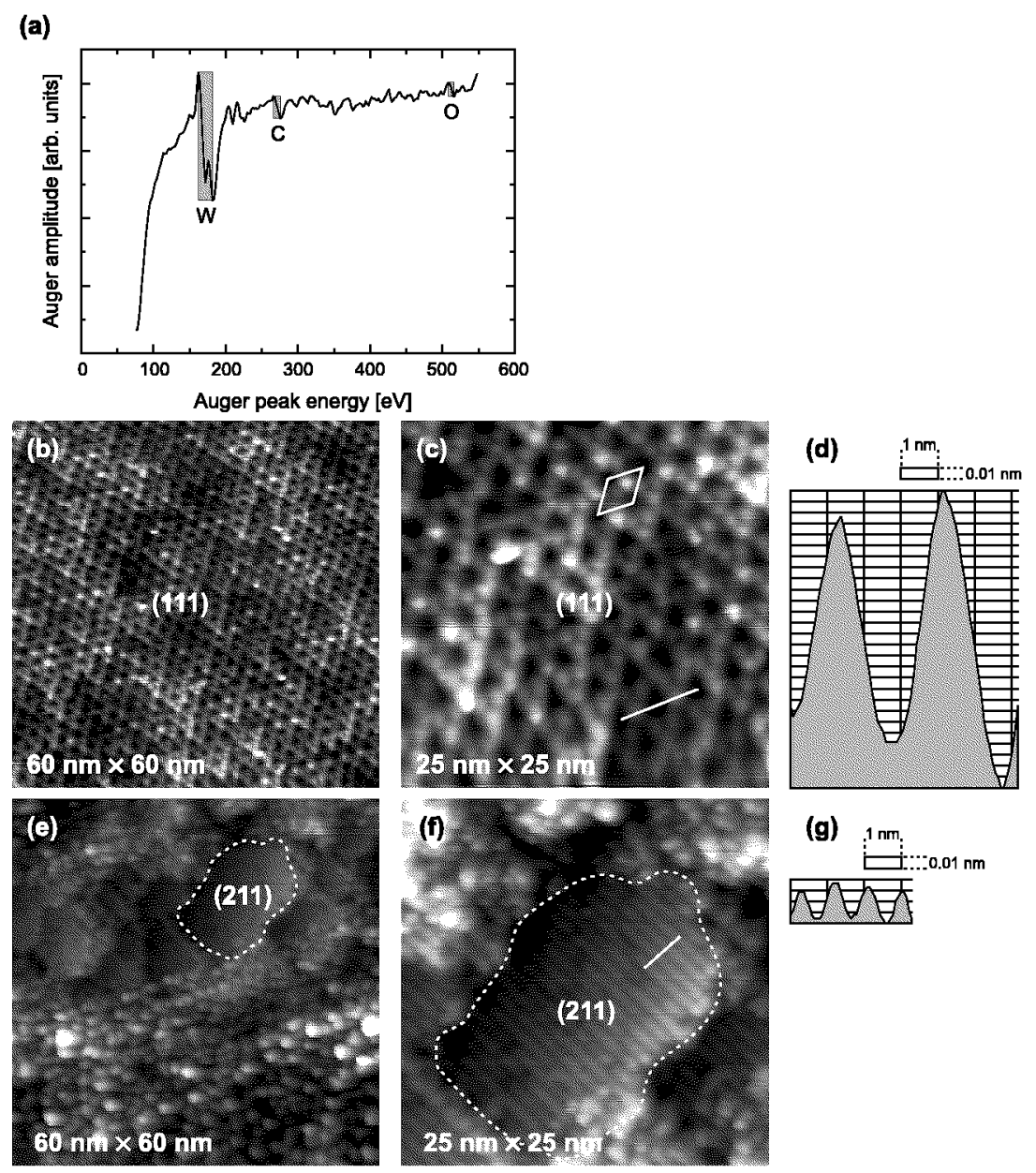

(g) $1{ }^{1 \mathrm{~nm}}: \ldots .01 \mathrm{~nm}$

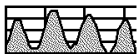

Fig. 1. (a) The Auger spectrum of the tungsten sample. (b) STM image of reconstructed W(111) surface. (c) The same surface at a higher magnification. The white rhombus indicates an elementary cell of the reconstruction pattern. (d) A height profile along the segment indicated in (c). (e)-(f) A region of reconstructed W(211) facets. (g) A height profile along the segment indicated in (f).

In conclusion, we found, using high resolution real-space STM imaging, that a submonolayer coverage of carbon and oxygen causes a $6 \times 6$ reconstruction of $\mathrm{W}(111)$ surface and a $2 \times n(n=1$ or 2$)$ reconstruction of $\mathrm{W}(211)$ surface.

\section{Acknowledgments}

This work has been supported by University of Wrocław, grant No. 2016/W/IFD. 


\section{References}

[1] C.-H. Nien, T.E. Madey, Surf. Sci. Lett. 380, L527 (1997).

[2] C.-H. Nien, T.E. Madey. Y.W. Tai, T.C. Leung, J.G. Che, C.T. Chan, Phys. Rev. $B$ 59, 10335 (1999).

[3] J. Kolaczkiewicz, E. Bauer, Surf. Sci. 420, 157 (1999).

[4] J.J. Kolodziej, T.E. Madey, J.W. Keister, J.E. Rowe, Phys. Rev. B 65, 075413 (2002).

[5] C.-H. Nien, T.E. Madey, Surf. Sci. 433-435, 254 (1999).

[6] www.nanotec.es.

[7] Y. Kuk, STM on Metals, in: Scanning Tunneling Microscopy I, Eds. H.-J. Güntherodt, R. Wiesendanger, Springer-Verlag, Berlin 1992.

[8] Y.B. Losovyj, A. Ciszewski, S. Zuber, Phys. Status Solidi B 225, R6 (2001). 